\title{
The Diagnostic and Prognostic Impact of Serum miRNA-21 in a Sample of Hepatitis C/None Hepatitis Diffuse Large B Cell Lymphoma Egyptian Patients
}

\author{
Nabil El-Halawani' ${ }^{1}$, Aida Nazir'1, Nagwa Mashali², Amani Sorour ${ }^{3}$, Mayada A. Moussa ${ }^{*}$ \\ ${ }^{1}$ Department of Haematology, Faculty of Medicine, Alexandria University, Alexandria, Egypt \\ ${ }^{2}$ Department of Pathology, Faculty of Medicine, Alexandria University, Alexandria, Egypt \\ ${ }^{3}$ Department of Clinical Pathology, Faculty of Medicine, Alexandria University, Alexandria, Egypt \\ Email: *drmayadaam@live.com
}

How to cite this paper: El-Halawani, N., Nazir, A., Mashali, N., Sorour, A. and Moussa, M.A. (2017) The Diagnostic and Prognostic Impact of Serum miRNA-21 in a Sample of Hepatitis C/None Hepatitis Diffuse Large B Cell Lymphoma Egyptian Patients. American Journal of Molecular Biology, 7 , 49-72.

http://dx.doi.org/10.4236/ajmb.2017.71005

Received: December 16, 2016

Accepted: January 15, 2017

Published: January 18, 2017

Copyright $\odot 2017$ by authors and Scientific Research Publishing Inc. This work is licensed under the Creative Commons Attribution-NonCommercial International License (CC BY-NC 4.0). http://creativecommons.org/licenses/by-nc/4.0/ (c) (i) (8) Open Access

\begin{abstract}
Background: Circulating microRNAs are potential biomarkers of diagnostic and prognostic impact in various inflammatory and malignant diseases. Aim: Linking inflammation with malignancy, we studied miRNA-21 in sera of hepatitis-C-virus (HCV) and none hepatitis diffuse large B-cell lymphoma (DLBCL) patients, aiming to identify its differential expression and prognosis in DLBCL with its subtypes; germinal center B-cell (GCB) and activated B-cell-like (ABC) and to evaluate its relation with HCV. Subjects and Methods: MiRNA-21 expression was measured using TaqMan quantitative RT-PCR in sera of 30 newly diagnosed DLBCL patients (HCV positive $(\mathrm{n}=10), \mathrm{HCV}$ negative $(\mathrm{n}=20)$ ) and 20 controls ( $\mathrm{HCV}$ positive $(\mathrm{n}=10), \mathrm{HCV}$ negative $(\mathrm{n}=10)$ ). Results: MiRNA-21 expression was significantly higher in DLBCL patients than in control $(p=0.00)$. Significant positive correlations between miRNA21 and LDH, IPI and disease stage were detected $(\mathrm{p}<0.05)$. Significantly higher miRNA-21 was detected in ABC sub-type compared to GCB sub-type $(\mathrm{p}=0.00)$. Higher miRNA-21 was associated with worse response $(\mathrm{p}=0.016), 2$ years overall $(\mathrm{p}=0.017)$ and progression free survival with statistical significance $(\mathrm{p}=0.003)$. Significantly higher miRNA-21 levels were detected in HCV positive DLBCL patients compared to HCV negative patients $(\mathrm{p}=0.00)$. Higher miRNA-21 levels were detected in HCV positive ABC subtype than GCB subtype $(p=0.05)$. Significantly higher levels were also detected in HCV positive controls compared to HCV negative controls. Conclusion: Our study shows that miRNA-21 is over expressed in our patients with DLBCL, displaying higher levels in ABC than in GCB subtypes. MiRNA-21 is associated with poor response to treatment and survival in DLBCL. MiRNA-21 is a potential marker of necro-inflammation independent of its role in tumorogenesis, showing higher expression in HCV positive DLBCL patients compared to none hepatitis patients.
\end{abstract}




\section{Keywords}

Diffuse Large B-Cell Lymphoma, microRNA-21, Hepatitis C Virus, Germinal Center-B, Activated B-Cell-Like

\section{Introduction}

MicroRNAs are small RNA molecules which control the expression of many target messenger RNAs involved in cell differentiation, proliferation and apoptosis. Their aberrant expression is related to the pathogenesis of many human diseases such as infections, inflammatory disorders, immune diseases and cancers [1]. A single mi-RNA can potentially control many targets to provide coordinated regulation of a network of proteins in a particular tissue or at a specific developmental stage. However, miRNAs do not encode a protein product, but instead they act as post-transcriptional regulators, causing silencing of the target messenger RNA either by promoting its degradation or by blocking its translation [2].

The potential function of miRNAs in lymphomagenesis has been studied by evaluating the consequences of their up- or down-regulated expression. Depending on the target genes, miRNAs may act as potential oncogenes or as tumor suppressor genes depending on the cellular context in which they are expressed [1]. One of the first miRNAs to be discovered in human cells is miRNA-21, which was found to be overexpressed in a variety of diseases including inflammation, infections, immune diseases and most cancer types, both solid tumors and hematological malignancies [3]. It has been recognized as an anti-apoptotic oncomir. In most cancer patients high levels of miRNA-21 are associated with tumor aggressiveness as well as poor survival [4] [5] [6]. Elevated levels of miRNA-21 have been detected in sera and plasma of patients with different malignancies [7].

Several studies have addressed the expression and the significance of miRNA-21 in diffuse large B-cell lymphoma (DLBCL). Higher expression levels of miRNA-21 were detected in biopsy material [8] [9] as well as in sera of patients with DLBCL [9] [10] when compared to normal control. Significantly higher levels were detected in the poor risk activated B-cell like (ABC-like) subtype compared to the germinal center B (GCB) subtype [8]. The mechanism by which miRNA-21 contributes to pathogenesis of diffuse large B-cell lymphoma is still unclear. Among the many possible targets of miRNA-21 the most relevant in pathogenesis of DLBCL are phosphatase and tensin homolog (PTEN) and forkhead box protein O1 (FOXO1). It was recently suggested that miR-21 might modulate the PI3K/AKT pathway by targeting PTEN upstream and FOXO1 downstream [11]. In addition, miRNA-21 expression was correlated with chemoresistance in DLBCL cells through the up-regulation of multi-drug resistance (MDR1) expression and activity which is mediated by suppression of PTEN and activation of AKT pathway [12].

Hepatitis C virus (HCV) infection is a global health problem. About 170 - 200 million people, approximately $3 \%$ of the world's population are chronically infected with HCV [13] and at risk of developing liver disease including liver cirrhosis and hepato- 
cellular carcinoma and B-cell lymphoma [14]. Several circulating miRNAs established their potential value as biomarkers for detection and prediction of liver disease progression in HCV infection [15]. MiRNA-21 is one of the most important miRNAs prevalently expressed in $\mathrm{HCV}$ positive patients.

Unlike all other malignancies, studies of the prognostic implication of miRNA-21 expression in patients with DLBCL have been a matter of debate. Multiple studies revealed that higher levels of miRNA-21 were associated with better prognosis in DLBCL [8] [9] [10], while others suggested a poor prognostic impact of miRNA-21 [16]. In addition to our knowledge, there are no existing data up to date on the expression of miRNA-21 in HCV related DLBCL. Most of the studies conducted were performed on splenic marginal zone lymphoma (SMZL) patients [17]. Thus in this study we aimed to identify the expression and the prognostic implications of miRNA-21 in DLBCL cases and to evaluate its differential expression in $\mathrm{GCB}$ and $\mathrm{ABC}$ sub-types and its impact on response to chemotherapy as well as survival in each sub-type. In addition, our study also intended to illustrate the relation between miRNA-21 and HCV infection in DLBCL and non-lymphoma patients.

\section{Material and Methods}

This study included thirty patients with newly diagnosed diffuse large B cell lymphoma admitted to the hematology unit at Alexandria Main University Hospital. The diagnosis of DLBCL was done by applying the criteria of the WHO classification of tumors of the hematopoietic and lymphoid tissues 2008 and further confirmed by histopathological examination and immunohistochemical analysis. Twenty subjects without lymphoma of matched age and sex were included in this study as a control group. The samples were collected over a period of 18 months starting from May 2013 till October 2014. The patients were further followed up for a period of 24 months from the time of sample collection. The laboratory investigations, bone marrow trephine biopsy and miRNA studies were performed at the clinical pathology department, while histopathological examination of tissue specimens was carried out at the pathology department in the same hospital. The studied patients $(n=30)$ aged 20 to 68 years of age with a mean of $52.13 \pm 12.12$ years. Among the studied patients 17 were males $(56.7 \%)$ and 13 females (43.3\%) with a male to female ratio of $1.3: 1$. They were all pathologically confirmed newly diagnosed DLBCL cases with an ECOG PS of no more than score 2. The age of control group $(\mathrm{n}=20)$ ranged from 29 to 68 years of age with a mean of $47.4 \pm 11.31$. Eleven were males (55\%) and nine were females (45\%) with a male:female ratio of 1.2:1. All the subjects included in the study were subjected to estimation of Anti-HCV antibodies serology by Enzyme Linked Immunosorbant Assay (ELISA) technique at the start of the study. We used HCV antibodies ELISA kit (Abnova). Those subjects with positive serological tests for Anti HCV antibodies were further submitted to HCV genetic detection and quantification by polymerase chain reaction (PCR). HCV RNA Quantification Assay Roche COBAS ${ }^{\circledast}$ Ampliprep Total Nucleic Acid Isolation Kit (TNAI) was used. The patient group was further classified into; 10 DLBCL patients with HCV infection and 20 DLBCL patients without HCV infection. Similarly, the control group was classified into 10 completely healthy subjects and 10 subjects without 
lymphoma but with HCV infection (HCV pathological control).

Biopsy material was obtained from DLBCL patients before treatment from one or more sites, including: lymph nodes, extra nodal masses or spleen. Excisional biopsy was performed in 25 patients. (83.33\%), which included; excisional biopsy of clinically detected lymph nodes in 23 cases $(76.67 \%)$, splenectomy in one case $(3.33 \%)$ and right hemi-colectomy in one case $(3.33 \%)$. CT guided core biopsy from abdominal lymph node/mass was performed in 4 cases (13.33\%). CT guided core biopsy from anterior mediastinal mass was done in one case (3.33\%). BM trephine biopsy was performed in all cases $(n=30)$ due to its importance in staging of the patients. Radiological assessment in the form of CT neck, chest, abdomen and pelvis was performed initially for all DLBCL patients at diagnosis and for follow up after 4 cycles of chemotherapy and at the end of therapy.

All the patients received the standard CHOP-21 (Cyclophosphamide, Hydroxydaunorubicin, oncovin and prednisolone) protocol and were followed up and first re-assessed after 4 cycles. Patients were evaluated in view of the response criteria of lymphoma. Those patients who achieved objective response (CR/PR) further received CHOP protocol for a total of $6-8$ cycles, while those who showed no response received 2 - 3 cycles of salvage chemotherapy protocols; DHAP or ESHAP protocols and were planned for autologous BMT. End of therapy assessment was performed for all patients. All the patients were evaluated for chemotherapy toxicity based on WHO toxicity grades. The patients were followed up for an average of 24 months. Survival analysis was performed. Overall survival (OS) and progression free survival (PFS) were calculated for all DLBCL patients.

The HCV positive controls were evaluated at the start of the study for the severity of hepatic condition using laboratory investigations including liver enzymes, serum albumin, total and direct bilirubin, prothrombin activity and viral load, as well as radiological examination by ultrasonographic study.

The study was approved by the Ethics Committee of Alexandria Faculty of Medicine. Ethical permission and informed consent were obtained from all participants.

\subsection{Immunohistochemistry}

Hematoxylin- and eosin-stained sections were prepared from the paraffin-embedded tissue blocks. Formalin-fixed paraffin sections of $3 \mu \mathrm{m}$ thick were used for IHC staining. Staining of tissue sections with a routine panel of antibodies to $\mathrm{CD} 3(\mathrm{CD} 3 \varepsilon \mathrm{Ab}-2$, Clone PS1, Mouse Monoclonal Antibody, Thermo Fisher Scientific. UK), CD20 (CD20 Ab-1, Clone L26, Mouse Monoclonal Antibody Thermo Fisher Scientific, UK), CD15 (CD15 Ab-3, Clone MMA; same as LeuM1, Mouse Monoclonal Antibody Thermo Fisher Scientific, UK) and CD30 (CD30 Ab-1, Clone Ber-H2, Mouse Monoclonal Antibody Thermo Fisher Scientific, UK) was done.

The diagnosis of DLBCL and its sub-classification in GCB and ABC subtypes were done by applying the criteria of the WHO classification of tumors of the hematopoietic and lymphoid tissues 2008 [18] and revised in 2016 [19] and were confirmed by IHC using the following antibodies; Antibodies to CD10 (CD10/CALLA (Neural Endopeptidase) Ab-2, Clone 56C6 Mouse Monoclonal Antibody Thermo Fisher Scientific, UK), BCL-6 (Clone BL6.02 PG-B6p, Mouse Monoclonal Antibody Thermo Fisher Scientific, 
UK), MUM-1 (IRF4-MUM1p; Mouse Monoclonal Antibody Thermo Fisher Scientific, UK) and BCL-2 (bcl2 alpha AB-1, Clone 100/D5, Mouse Monoclonal Antibody Thermo Fisher Scientific, UK), were used for the staining.

All the immunostained slides were assessed using visual estimation to determine the percentage of tumor cells having a positive reaction. CD10 and BCL-2 were considered to be expressed by the tumor if $>30 \%$ of tumor cells had positive surface membrane. Positive expression of MUM- 1 and BCL- 6 protein was considered when $>30 \%$ the tumor cells had positive immunoreactivity in the nuclei. Hans's algorithm [20] was used for the allocation of patients into two IHC sub-groups. Based on this algorithm cases were allocated to the GCB sub-group if $\mathrm{CD} 10$ alone was positive. $\mathrm{CD} 10$ negative cases were further tested for BCL- 6 . If BCL- 6 was negative, then the case was assigned to the $A B C$ sub-group. Cases with BCL-6 positivity were further stained with MUM1. If MUM1 was negative, then the case was assigned to the GCB sub-group while positive cases were allocated to the $\mathrm{ABC}$ sub-group.

Quantification of miRNA-21

MiRNA-21 detection and quantification in serum of DLBCL patients as well as the control group by Real Time Reverse Transcriptase Polymerase Chain Reaction (RTPCR) was performed initially at diagnosis. MiRNA-24 was used as an endogenous control for miRNA-21.

\subsection{Extraction of Total RNA}

Total RNA, including miRNA, isolation from peripheral blood samples was carried out with the miRNeasy Mini Kit (QIAGEN, Maryland, USA) according to the manufacturer's instructions. The concentration and purity of RNA were measured at 260, 280 and $230 \mathrm{~nm}$ using NanoDrop2000 Spectrophotometer (Thermo Scientific, USA). A260: A230 ratio greater than 1.7 and A260:A280 ratio greater than 2.0 indicates highly pure RNA.

\subsection{Detection and Quantification of miRNA}

Detection and quantification of miRNA was performed using TaqMan ${ }^{\varpi}$ MicroRNA Assays-Applied Biosystems real-time PCR instruments. Gene Expression Assays were performed in a two-step; Reverse transcription (RT) step, in which cDNA is reverse transcribed from the extracted RNA using miRNA-21 and miRNA-24 specific primers according to Taqman MicroRNA assay protocol. We used the TaqMan ${ }^{\oplus}$ MicroRNA Reverse Transcription kit (Applied Biosystems, USA). Reverse transcription was carried out in $15 \mu$ reaction contained $10 \mathrm{ng}$ of RNA samples. The reactions were incubated in (Biometrathermocycler) for $30 \mathrm{~min}$ at $16^{\circ} \mathrm{C}, 30 \mathrm{~min}$ at $42^{\circ} \mathrm{C}, 5 \mathrm{~min}$ at $85^{\circ} \mathrm{C}$ and then held at $4^{\circ} \mathrm{C}$. This is followed by the PCR amplification step in which PCR products are quantitatively synthesized from cDNA using the Taqman ${ }^{\circledR}$ Universal PCR Master Mix II. Real time PCR was performed in $20 \mu \mathrm{l}$ reaction mixture included $1.33 \mu \mathrm{l}$ R product, $10 \mu \mathrm{l}$ TaqMan Universal PCR Master Mix II, $1 \mu \mathrm{l}$ of primers and probe mix and $7.67 \mu \mathrm{l}$ nuclease-free water according to TaqMan MicroRNA Assay protocol (PE, Applied Biosystems). Reactions were incubated at $95^{\circ} \mathrm{C}$ for $10 \mathrm{~min}$, followed by 40 cycles of $95^{\circ} \mathrm{C}$ for $15 \mathrm{~s}$ and $60^{\circ} \mathrm{C}$ for $10 \mathrm{~min}$. MiRNA-24 was used as an endogenous reference for 
normalizing the expression levels of miRNA-21. Data processing was performed using Rotor Gene Q 3000 software.

\subsection{Data Analysis}

The threshold cycle data $(\mathrm{Ct})$ and baselines were determined using auto settings. The relative quantification of miRNA-21 expression was calculated using the comparative $\mathrm{C}_{\mathrm{T}}$ method $\left(2^{-\Delta \Delta \mathrm{CT}}\right) . \Delta \mathrm{CT}$ is the difference of $\mathrm{CT}$ value between the target (miRNA-21) and endogenous reference (miRNA-24); ( $\triangle \mathrm{CT}=\mathrm{CT}$ miRNA-21 - CT miRNA-24). $\Delta \Delta \mathrm{Ct}$ is the difference of the $\Delta \mathrm{Ct}$ value between the sample and a value calibrator constant (in this case we used the mean of $\Delta \mathrm{Ct}$ of normal HCV negative healthy controls); $(\Delta \Delta \mathrm{CT}=\Delta \mathrm{CT}$ miRNA sample- $\Delta \mathrm{CT}$ control miRNA).

The relative expression ratio of miRNA-21 was presented as the fold change normalized to reference miRNA-24 and relative to the normal control subject.

If expression level of the sample miRNA-21 is equal to that of the normal healthy control subject, then the $\Delta \Delta \mathrm{C}_{\mathrm{t}}$ equals zero and $2^{-\Delta \Delta \mathrm{CT}}$ equals one. Therefore, by comparing the value of $\Delta \mathrm{C}_{\mathrm{t}}$ of sample miRNA-21 and $\Delta \mathrm{C}_{\mathrm{t}}$ normal control subject miRNA-21, we could compare the expression level of miRNA-21 in the patients with normal control subject.

If the fold-change is $>1$, then the result was reported as a fold up-regulation. If the fold-change is $<1$, then the negative inverse of the result was reported as a fold downregulation.

\subsection{Statistical Analysis}

Data were analyzed using the Statistical Package for Social Sciences (SPSS ver.20 Chicago, IL, USA). The given graphs were constructed using the same program as well as Microsoft Excel software. The distributions of quantitative variables were tested for normality using Kolmogorov-Smirnov test, which revealed that the data are not normally distributed. Quantitative data were described using median, range. Qualitative data were described using number and percent.

Correlations between quantitative variables were done using Spearman rank correlation test. Comparing quantitative variables between 2 groups was conducted using Mann Whitney $U$ test. Comparing quantitative variables between 3 groups was conducted using Kruskal Wallis test. Comparing $2 \times 2$ categorical variables was done using Pearson Chi square test, while Fisher's exact test (FEP) was used when $>20 \%$ of cells have expected cell count of less than 5. Monte Carlo significance test (MCP) was used in $>2 \times 2$ table when $>20 \%$ of cells have expected cell count less than 5 .

Kaplan Meier survival analysis was carried out for overall survival and progression free survival time of DLBCL cases. Overall survival was calculated from the time of inclusion in the study till the date of death, PFS was calculated from the time of inclusion in the study till the date of progression or death. Patients who were alive progression free at the time of last contact were censored for analysis. Log rank test was used to compare survival between low and high miRNA-21 (median value of cases was used as a cutoff point). In all statistical tests, level of significance of 0.05 was used, below which the results were considered to be statistically significant. 


\section{Results}

This study was carried out on 30 newly diagnosed subjects with DLBCL and 20 subjects of matched age and sex as control group. Among the lymphoma patients, twenty patients were negative for $\mathrm{HCV}$ infection (66.7\%) while the remaining ten were positive for HCV infection (33.3\%).

\subsection{Histopathology}

Microscopic description; Centroblastic morphology was identified in 12 cases (40\%) of which 10 cases had a monomorphic pattern and 2 cases had a polymorphic pattern. The ABC sub-type included; 8 cases $(26.67 \%)$ of the immunoblastic sub-type and 4 cases (13.3\%) of the anaplastic type with Reed Sternberg like cells. A polymorphic pattern was detected in additional 4 cases (13.33\%); including 2 cases with plasmacytoid features. The last 2 cases had a morphology of Burkitt like large cell lymphoma (6.67\%).

Twelve cases (40\%) were assigned as GCB-DLBCL, while the other 18 cases $(60 \%)$ were ascribed as ABC-DLBCL. This diagnosis was based on both morphological features and immunohistochemical staining of the paraffin sections, using CD10, BCL6 and MUM1 antibodies. According to expression of CD10 of $>30 \%$, BCL6 of $>30 \%$ and MUM1 of $>30 \%$ the two histologically recognized sub-types could be confirmed.

In the GCB cases $(n=12)$, CD10 was expressed in 8 cases $(66.67 \%)$ and was absent in 4 cases $(33.33 \%)$. BCL6 was positive in 9 cases (75\%) and negative in 3 cases (25\%). None of the GCB subtype was positive for MUM1. BCL2 expression was positive in 8 cases $(66.67 \%)$ and negative in 4 cases $(33.33 \%)$ (Figure 1 ).

In the $\mathrm{ABC}$ like cases $(\mathrm{n}=18)$, none of the cases were positive for CD10 (0\%). BCL6 was positive in 3 cases (16.67) and negative in 15 cases (83.33\%). MUM1 was positive in 12 cases $(66.67 \%)$ and negative in 6 cases $(33.33 \%)$. BCL2 was expressed in 12 cases $(66.67 \%)$ and was absent in 6 cases (33.33\%) (Figure 2).

\subsection{International Prognostic Scoring System, Performance Status and Staging of DLBCL Patients}

Twelve cases (40\%) had an ECOG score of 0 - 1, while 18 cases (60\%) has an ECOG score of $\geq 2$. Fourteen DLBCL patients (46.67\%) were in early stage (I/II), while 16 cases (53.33\%) were in advanced stage (III/IV). Distribution of patients based on ECOG PS, the IPI and Ann Arbor staging system are summarized in Table 1.

\subsection{Chemotherapy and Response to Treatment}

Twenty nine patients (96.67\%) received 4 cycles of CHOP and were re-assessed both clinically and radiologically thereafter. One case died early in the study received a total of two cycles of CHOP.

Based on clinical examination, results of CT scans after the fourth cycle of CHOP and criteria of response; 10 patients (33.3\%) received a total of 6 cycles of CHOP. 11 patients $(36.67 \%)$ received a total of 8 cycles of CHOP. For those patients who showed no response (SD/PD) after the $4^{\text {th }}$ cycle of CHOP, 2 - 3 cycles of salvage chemotherapy protocols were used; 4 cases received ESHAP protocol (13.33\%), while the remaining four received DHAP protocol (13.33\%). 


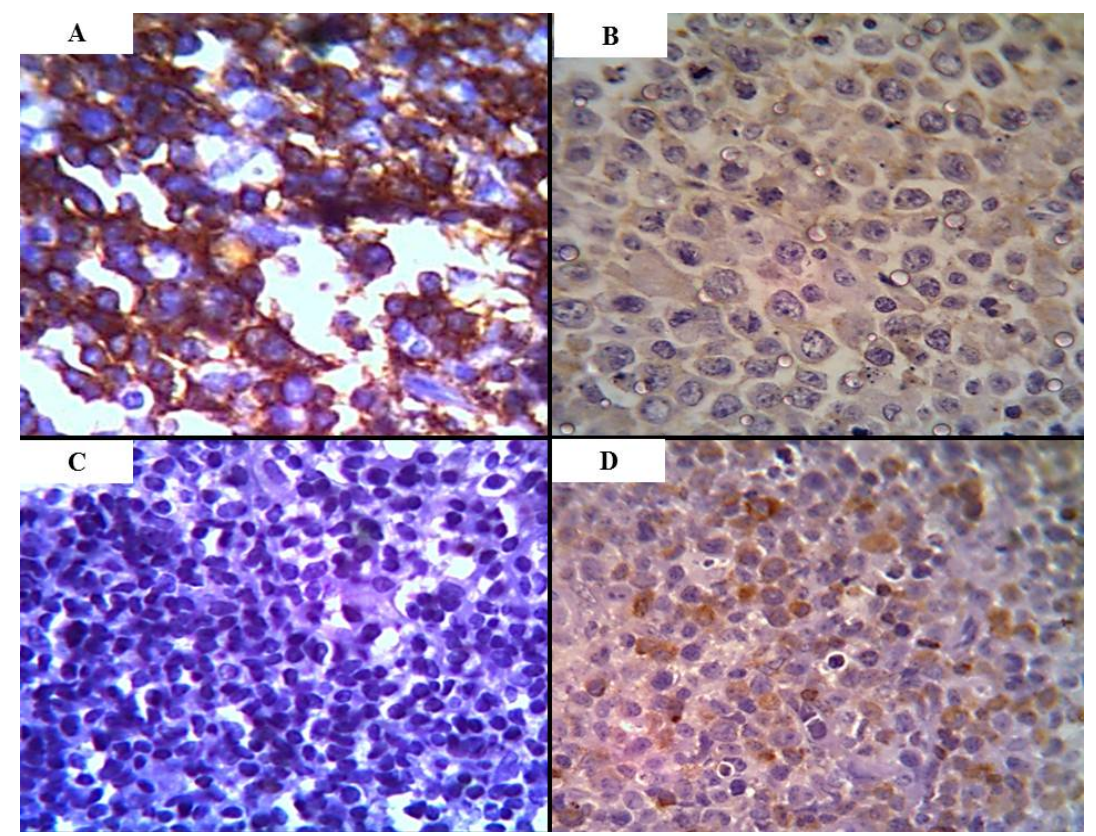

Figure 1. Diffuse large B cell lymphoma; Germinal center B cell phenotype (GCB-DLBCL). Excisional biopsy of the lymph node showing complete loss of architecture with replacement by a neoplastic cellular population composed of diffuse sheets of predominantly large cells with large vesicular nuclei, prominent membrane bound nucleoli and indistinct cytoplasm. Mitotic figures are readily detected. (A) Strong cell membrane positive staining reaction for CD20. (B) Cell membrane positive staining for CD10. (C) Negative staining reaction of all the populations of cells for BCL6. (D) Strongnuclear staining reaction for Bcl2.

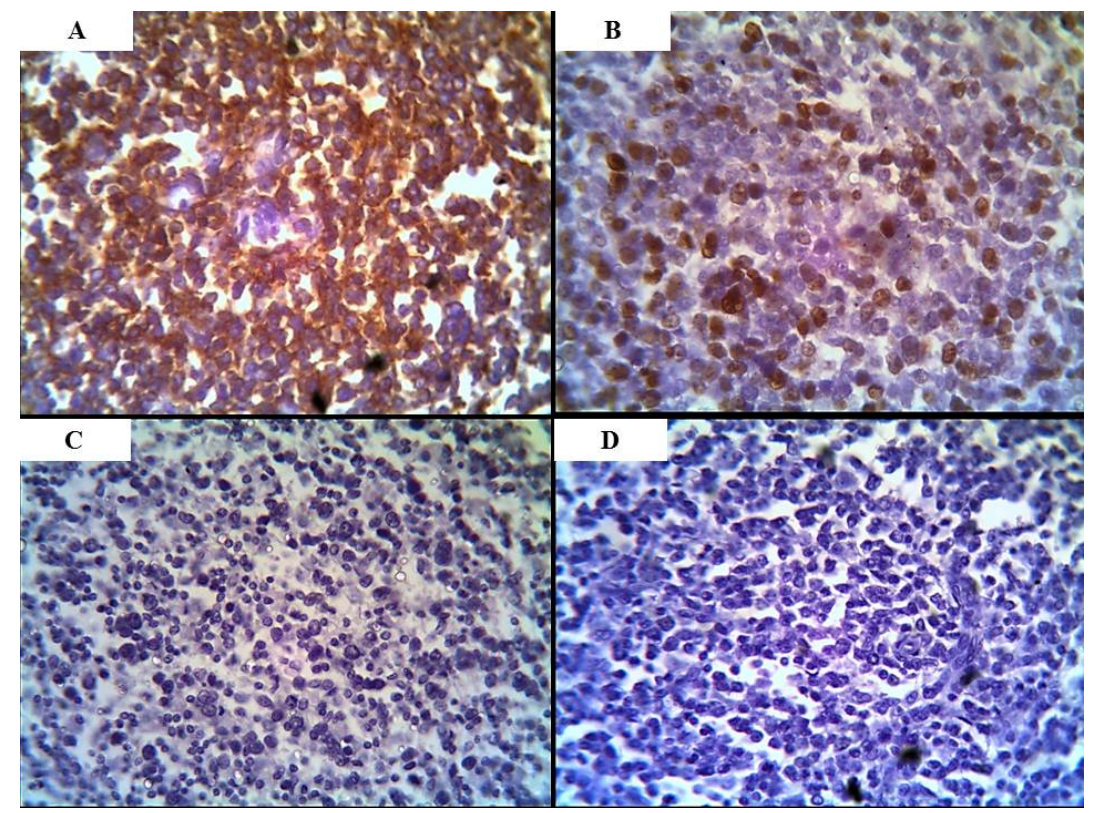

Figure 2. Diffuse large B cell lymphoma; Activated B cell phenotype (ABC-DLBCL). Excisional biopsy of the lymph node showing complete loss of architecture with replacement by a neoplastic cellular population composed of diffuse sheets of large cells with large vesicular nuclei, prominent nucleoli and variable amount of cytoplasm. Mitotic figures are readily detected. (A) Strong cell membrane positive staining reaction for CD20. (B) Moderate nuclear staining of about $30 \%$ of the cells for MUM1. (C) Negative cell membrane staining reaction of all the populations of cells for CD10. (D) Negative nuclear staining reaction for BCL6. 
Table 1. Performance status, staging and prognostic scoring system of the studied DLBCL patients $(\mathrm{n}=30)$.

\begin{tabular}{ccc}
\hline & No. & $\%$ \\
\hline ECOG PS $(\mathrm{n}=30)$ & 3 & 10 \\
0 & 9 & 30 \\
1 & 18 & 60 \\
$\geq 2$ & & \\
Ann Arbor Staging system $(\mathrm{n}=30)$ & 5 & 16.67 \\
I & 9 & 30 \\
II & 8 & 26.67 \\
III & 8 & 26.67 \\
IV & 8 & \\
IPI of DLBCL patients $(\mathrm{n}=30)$ & & 13.33 \\
0 & 4 & 16.67 \\
1 & 5 & 16.67 \\
2 & 5 & 30 \\
3 & 9 & 10.33 \\
4 & 4 & \\
5 & & \\
\hline
\end{tabular}

At the end of therapy 29 cases of DLBCL were evaluated both clinically and radiologically. Twelve cases (41.38\%) entered in CR, while seven cases (24.14\%) achieved PR, with an OR (CR + PR) of 65.52\%. Ten cases (34.48\%) showed no response (SD/PD).

In the GCB subtypes $7 / 12$ cases (58.33\%) entered in CR, while $2 / 12$ cases (16.67\%) achieved PR with an OR of $75 \%$. Three cases (25\%) showed no response (SD/PD). In the ABC subtype $5 / 17$ cases (29.41\%) achieved CR, while $5 / 17$ cases (29.41\%) achieved PR, with an OR of $58.82 \%$. Seven cases (41.18\%) showed no response.

\subsection{HCV Positive DLBCL Cases}

HCV infection was diagnosed in 10 DLBCL patients (33.3\%), by serological as well as molecular tests. HCV infection was more commonly detected in ABC DLBCL ( $\mathrm{n}=$ $7 / 10,70 \%)$ than in GCB subtype $(\mathrm{n}=3 / 10,30 \%)$. All $\mathrm{HCV}$ positive patients received $\mathrm{CHOP}$ protocol $(100 \%)$. Pretreatment transaminases, total and direct bilirubin were elevated in 5 patients (50\%) among whom four patients had mild to moderate hepatic impairment and were managed with liver support and chemotherapy dose adjustment of doxyrubicin and/or etoposide and could tolerate chemotherapy well without worsening hepatic condition. The last case had obstructive jaundice and he was managed with steroids then with reduction of serum bilirubin and transaminases, he could safely start chemotherapy with dose adjustment. However, unfortunately, he progressed to grade 4 hepatic toxicity after 4 cycles of $\mathrm{CHOP}$ which indicated discontinuation of chemotherapy. 
Regarding response to treatment in HCV positive DLBCL patients, $4 / 10$ cases (40\%) entered in CR, $3 / 10$ cases (30\%) achieved PR with an OR of $70 \%$, while 3 cases (30\%) showed no response.

\subsection{Control Group}

Twenty subjects without lymphoma participated in the study as control, of which 10 were infected with $\mathrm{HCV}$ (50\%), while the remaining 10 were apparently healthy individuals (50\%). Table 2 shows data regarding age, sex and hepatic parameters in HCV positive DLBCL patients and HCV positive pathological control.

\subsection{Serum miRNA-21 Results}

- Serum miRNA-21 in DLBCL patients compared to control.

Serum miRNA-21 in the DLBCL patients $(n=30)$ ranged from 3.03 to 38.53 with a median of 9.32. While, in the control group miRNA-21 ranged from 0.7 to 22.47 with a median of 1.44. A highly statistically significant difference between miRNA-21 expression levels in the DLBCL compared to its levels in control group was detected $(\mathrm{U}=115$, $\mathrm{p}=0.00)$ (Figure 3).

- Serum miRNA-21 expression levels compared in DLBCL subtypes; GCB and $\mathrm{ABC}$.

In the GCB DLBCL $(n=12)$ serum miRNA-21 ranged from 3.03 to 11.63 with a

Table 2. Age, sex and different hepatic parameters in HCV positive DLBCL patients and HCV positive pathological control.

\begin{tabular}{ccc}
\hline Parameter & $\begin{array}{c}\text { HCV positive DLBCL patients } \\
(\mathrm{n}=10)\end{array}$ & $\begin{array}{c}\text { HCV positive controls }(\mathrm{n}=10) \\
\text { min-max }(\mathrm{mean} / \mathrm{SD})\end{array}$ \\
\hline Age (Years) & $38-68$ & $34-68$ \\
& $53.4 \pm 10.51$ & $(49 \pm 10.74)$ \\
Sex (M/F) & $(4 / 6)$ & $(6 / 4)$ \\
& $1: 1.5$ & $1.5: 1$ \\
AST(U/l) & $16-278$ & $20-250$ \\
& $70.2 \pm 77.44$ & $(84.2 \pm 86.85)$ \\
ALT(U/l) & $14-320$ & $23-480$ \\
& $92 \pm 89.95$ & $(124.6 \pm 147.36)$ \\
ALP(U/l) & $77-534$ & $62-347$ \\
& $175.9 \pm 142.52$ & $(138.6 \pm 85.96)$ \\
Total Bilirubin (mg/dl) & $1-18$ & $0.58-4$ \\
& $3.16 \pm 5.25$ & $(1.616 \pm 0.707)$ \\
Direct Bilirubin (mg/dl) & $0.1-16.5$ & $0.16-2.8$ \\
& $2.33 \pm 5$ & $(0.914 \pm 0.97)$ \\
Serum albumin (mg/dl) & $1.5-4$ & $2.6-4.2$ \\
& $3.25 \pm 0.74$ & $(3.43 \pm 0.53)$ \\
Prothrombin activity (\%) & $32-100$ & $50-95$ \\
& $81 \pm 0.21$ & $(78 \pm 0.156)$ \\
Viral load (IU) & $20,000-6,000,000$ & $50,004,800,000$ \\
& $1,151,000 \pm 1,777,279$ & $1,200 \pm 1,628,185$ \\
\hline
\end{tabular}




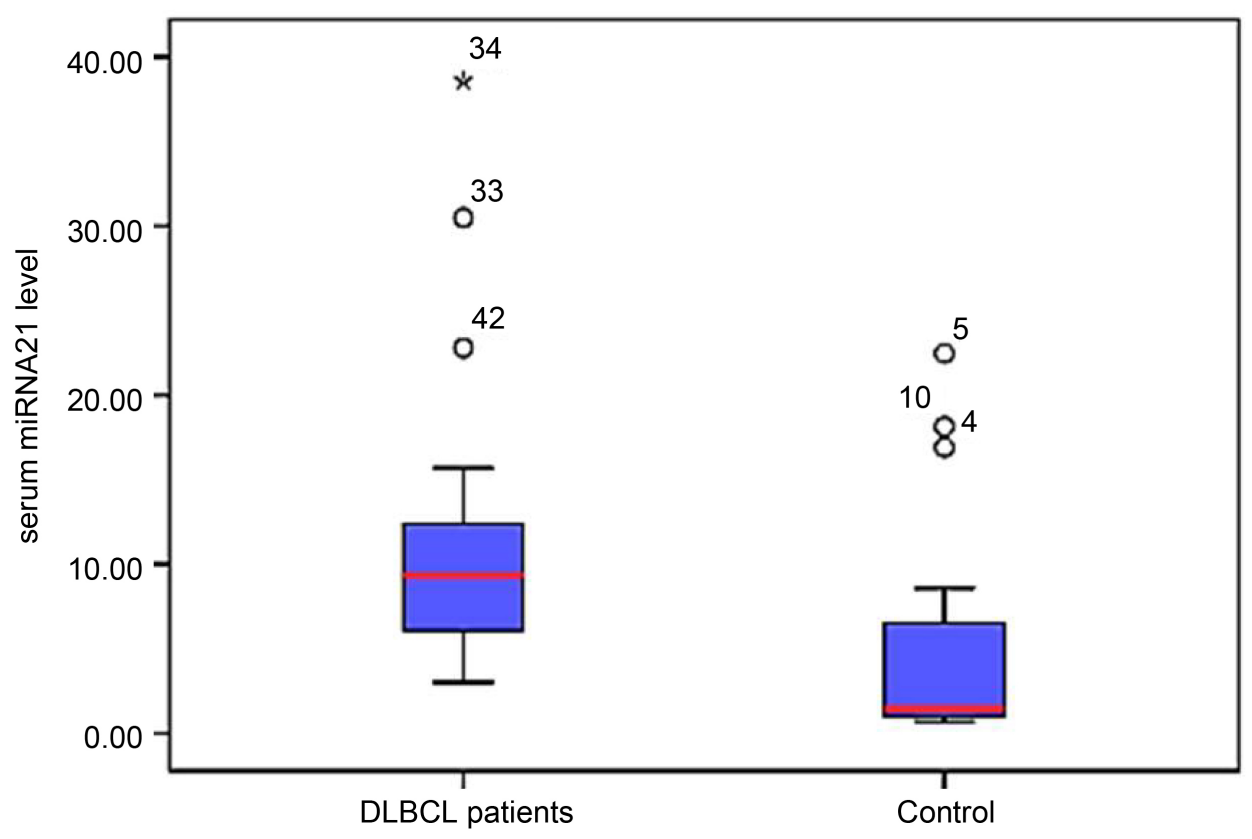

Figure 3. Serum miRNA-21 in DLBCL patients compared to controls. Serum miRNA-21 shows higher expression in DLBCL patients $(n=30)$ compared to controls $(n=20)$. In the box plots, the band inside the box is the median. The outlier's samples (those whose expression extends beyond 1.5 times the interquartile range) are represented as dots and stars: the white dots denotes the middle outlier while the black stars are the extreme outliers.

median of 5.67. While, in the ABC DLBCL $(\mathrm{n}=18)$ miRNA-21 ranged from 5.82 to 38.58 with a median of 11.44 . A significantly higher level of miRNA-21 was detected in ABC DLBCL cases compared to GCB cases $(U=24.00, p=0.00)$ (Figure 4).

- Correlations between serum miRNA-21 expression \& different parameters of clinical and prognostic significance in DLBCL cases.

Statistically significant positive correlations were detected between serum miRNA-21 and serum LDH $\left(r_{s}=0.454, p=0.012\right)$ the disease stage $\left(r_{s}=0.443, p=0.014\right)$ and the IPI $\left(r_{s}=0.395, p=0.031\right)$. While the correlations between miRNA-21 and the age $\left(r_{s}=\right.$ $0.231, \mathrm{p}=0.220)$, uric acid $\left(\mathrm{r}_{\mathrm{s}}=0.109, \mathrm{p}=0.567\right)$, ESR $\left(\mathrm{r}_{\mathrm{s}}=0.172, \mathrm{p}=0.363\right)$ and ECOG PS $\left(r_{s}=0.328, p=0.077\right)$ were not statistically significant (Table 3$)$.

- Comparing serum miRNA-21 levels in relation to expression of immunohistochemical markers of DLBCL.

Serum miRNA-21 was compared in relation to immunohistochemical markers of DLBCL. MiRNA-21 in the CD10 positive group $(\mathrm{n}=8)$ ranged from 3.03 to 10.02 (median; 4.95), while, in the CD10 negative group $(\mathrm{n}=22)$ miRNA-21 ranged from 5.28 to 38.58 (median; 11.195). CD10 negative cases showed significantly higher miRNA-21 expression than $\mathrm{CD} 10$ positive cases $(\mathrm{U}=21.00, \mathrm{p}=0.002)$.

BCL6 negative cases showed significantly higher miRNA-21 expression compared to cases positive for BCL6 $(\mathrm{U}=52.00, \mathrm{p}=0.018)$. Serum miRNA-21 in the BCL6 positive group $(n=12)$ ranged from 3.03 to 22.78 with a median of 6.105 , while, in the BCL6 negative group $(\mathrm{n}=18)$, miRNA-21 ranged from 3.41 to 38.58 with a median of 11.195. In the MUM1 positive group ( $\mathrm{n}=13$ ) miRNA-21 ranged from 5.82 to 38.58 with a median of 12.38. In MUM1 negative group $(n=17)$ miRNA-21 ranged from 3.03 to 13.83 


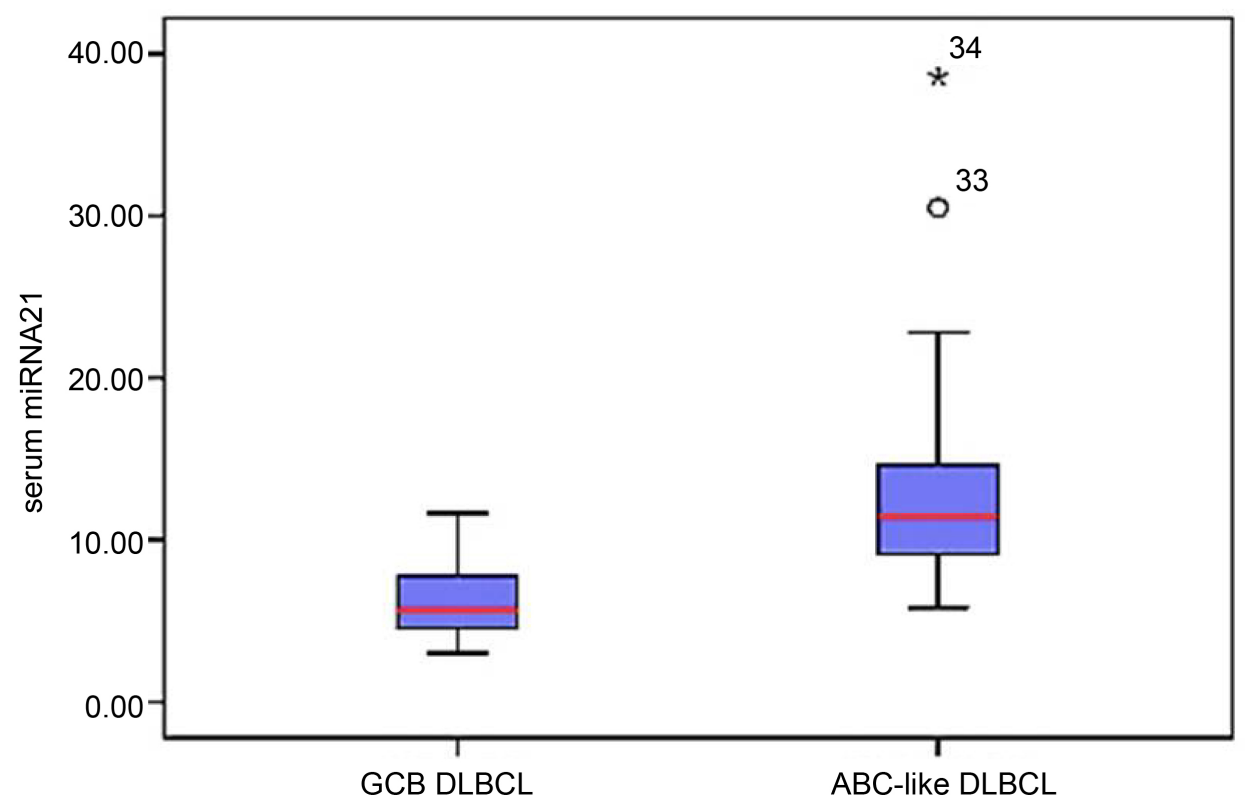

Figure 4. Comparing serum miRNA-21 levels in GCB versus ABC DLBCL patients. Serum miRNA-21 shows higher expression in ABC sub-type $(n=18)$ compared to GCB sub-type $(n=$ 12). In the box plots, the band inside the box is the median. The outlier's samples which extends beyond 1.5 times the interquartile range are shown as dots and stars: the white dots represent the middle outlier while the black stars are the extreme outliers.

Table 3. Correlations between serum miRNA-21 expression \& different parameters of clinical and prognostic significance in DLBCL cases.

\begin{tabular}{ccc}
\hline Parameter & \multicolumn{2}{c}{ Serum miRNA-21 DLBCL patients } \\
\hline & $\mathrm{r}_{\mathrm{s}}$ & $\mathrm{p}$ \\
\hline Age & 0.231 & 0.220 \\
LDH & 0.454 & $0.012^{*}$ \\
ESR & 0.172 & 0.363 \\
Uric acid & 0.109 & 0.567 \\
ECOG & 0.328 & 0.077 \\
Ann Arbor staging & 0.443 & $0.014^{*}$ \\
IPI & 0.395 & $0.031^{*}$ \\
\hline
\end{tabular}

$\mathrm{r}_{\mathrm{s}}$ : Spearman rho correlation coefficient. *: Significant correlation.

with a median of 7.06. MUM1 positive cases showed significantly higher miRNA-21 expression compared to MUM1 negative cases. $(\mathrm{U}=34.00, \mathrm{p}=0.001)$.

In cases positive for BCL2 $(n=20)$, miRNA-21 ranged from 4.29 to 38.58 with a median of 10.24, while, in the BCL2 negative group $(n=10)$ its level ranged from 3.03 to 14.62 with a median of 6.6050 . The result didn't reach statistical significance $(\mathrm{p}=$ 0.086).

- Serum miRNA-21 expression levels in DLBCL patients in relation to response to treatment.

Serum miRNA-21 in DLBCL patients who achieved CR $(n=12)$ ranged from 3.03 to 
13.83 with a median of 6.44 , while in patients who achieved $P R(n=7)$ it ranged from 5.28 to 22.78 with a median of 9.13. Patients who failed to respond to treatment had a serum miRNA-21 level that ranged from 6.06 to 38.58 with a median of 11.845. Higher miRNA-21expression levels were associated with poor response to treatment (Chi square $=8.244, \mathrm{p}=0.016)$ (Figure 5).

\section{- Differential expression of miRNA-21 in subtypes of DLBCL.}

Serum miRNA-21 level was compared in BCL2 positive and BCL2 negative GCB DLBCL cases, showing higher expression in the BCL2 positive group (median; 6.76) than in BCL2 negative group (median; 4.27) but the result didn't reach statistical significance $(\mathrm{U}=6.000, \mathrm{p}=0.089$ ). In ABC DLBCL cases, serum miRNA-21 in the BCL2 positive group (median; 11.845) was higher than its levels in BCL2 negative group (median; 9.485) but the result didn't reach statistical significance $(U=21.000, p=0.16)$.

In GCB DLBCL, the difference in miRNA-21 levels in cases with BM infiltration (median; 6.10) and those without BM infiltration (median; 5.20) were quite similar ( $\mathrm{U}=$ 8.000, $\mathrm{p}=0.667)$. In ABC-like cases the levels were higher in cases with BM infiltration (median; 14.95) when compared to cases without infiltration (median; 11.2), however the result didn't reach statistical significance $(U=14.000, p=0.137)$.

Serum miRNA-21 level in the GCB subtype with B symptoms and cases without B symptoms showed a median of 5.595 and 5.715 respectively, while in ABC-like patients with B symptoms, miRNA-21 levels (median; 13.3) were higher than in cases without B symptoms (median; 11.19). The difference in its expression regarding B symptoms in both subtypes didn't reach statistical significance $(G C B ; U=15.000, p=0.865, A B C ; U=$ 23.000, $\mathrm{p}=0.223$ ).

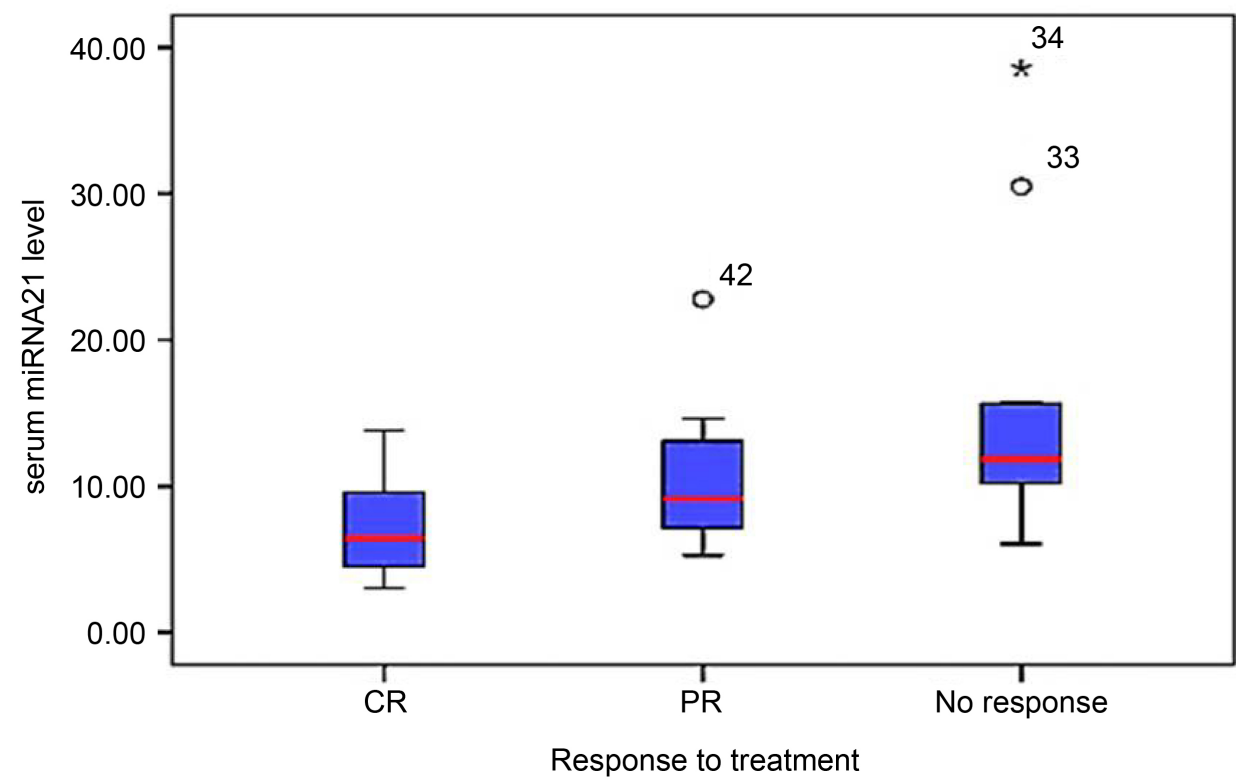

Figure 5. MiRNA-21 expression in relation to response to treatment in DLBCL patients Serum miRNA-21 expression levels were lowest in DLBCL patients who achieved CR $(n=12)$, followed by patients who achieved PR $(n=7)$, while patients who showed no response to chemotherapy $(\mathrm{n}=10)$ had the highest levels. The band inside each box plot is the median. The outlier's samples extending beyond 1.5 times the interquartilerange are illustrated as dots and stars: the white dots are the middle outlier while the black stars are the extreme outliers. 
MiRNA-21 levels were higher in GCB DLBCL cases with ECOG PS $\geq 2$ (median; 6.15) than in cases with ECOG 0-1 (median; 4.76), but the difference was not statistically significant $(\mathrm{U}=11.000, \mathrm{p}=0.291)$. Similarly, in ABC DLBCL cases, miRNA-21 expression levels were quite similar in cases with ECOG 0-1 (median; 11.08) and cases with ECOG $\geq 2$ (median; 12.38) $(\mathrm{U}=26.000, \mathrm{p}=0.258)$.

In GCB DLBCL cases with early stage (I/II), the median serum miRNA-21 was 5.13, while in cases with advanced stage (III/IV) the median was 6.15 with no significant difference $(U=13.000 \mathrm{p}=0.465)$. In ABC DLBCL cases, the difference in miRNA-21 expression between early stage (median; 10.295) and advanced stage (median; 14.42) was statistically significance $(\mathrm{U}=15.000, \mathrm{p}=0.026)$.

Correlations between serum miRNA-21 and the IPI in GCB $\left(r_{s}=0.408, p=0.188\right)$ and $\mathrm{ABC}\left(\mathrm{r}_{\mathrm{s}}=0.446, \mathrm{p}=0.064\right)$ sub-types were not statistically significant.

In GCB sub-group, in patients who achieved CR serum miRNA-21 showed a median of 4.76, while in patients with PR miRNA-21 had a median of 5.71. The highest levels were detected in patients who failed to respond to treatment (median; 7.46), but the result was not significant (chi square $=2275, \mathrm{p}=0.321$ ). In $\mathrm{ABC}$ sub-group, higher miRNA-21expression levels were associated with poor response to treatment (median; 14.22), compared to its levels in patients with PR (median; 11.56) and in patients who achieved CR (median; 7.41) with the result showing borderline statistical significance (chi square $=5.708, \mathrm{p}=0.058$ ).

\section{- MiRNA-21 expression in HCV positive versus HCV negative DLBCL patients.}

A Significantly higher serum miRNA-21 was detected in DLBCL patients with HCV infection ( $\mathrm{n}=10,8.11$ to 38.58 , median; 12.73 ) compared to patients without HCV infection ( $\mathrm{n}=20,3.03$ to 15.67 , median; 7.235) $(\mathrm{U}=26.00, \mathrm{p}=0.001)$.

- Comparing miRNA-21 expression levels in four groups; DLBCL HCV positive cases, DLBCL HCV negative cases, HCV positive control \& HCV negative control groups.

In HCV positive DLBCL cases $(\mathrm{n}=10)$ miRNA-21 showed the highest expression levels (8.11 to 38.58, median; 12.73). Lower levels were detected in HCV negative DLBCL cases $(\mathrm{n}=20,3.03$ to 15.67 , median; 7.235$)$ and in HCV positive control $(\mathrm{n}=$ $10,1.41$ to 22.47 , median; 6.515). In HCV negative control $(\mathrm{n}=10)$ miRNA-21 showed the lowest levels (0.7 to 1.87 , median; 1.0135). A highly statistically significant difference in miRNA-21 expression was detected between the four groups (chi square = 29.460, $\mathrm{p}=0.000$ ) (Figure 6).

- In HCV positive DLBCL, serum miRNA-21 was correlated with different parameters of prognostic significance, including:

IPI, stage, ECOG PS, Viral load, SGOT, SGPT, total and direct bilirubin, albumin and HCV prognostic score. Only the disease stage showed statistically significant correlation with miRNA-21 $\left(r_{s}=0.694\right.$, $\mathrm{p}$ value $\left.=0.026\right)$ while all other parameters; IPI, ECOG PS, viral load, total bilirubin, direct bilirubin, SGOT, and SGPT showed positive correlation with miRNA-21 level which failed to reach statistical significance. Serum albumin showed negative correlation with miRNA-21 which failed to reach statistical significance (Table 4).

- MiRNA-21 expression levels compared in HCV positive GCB and ABC patients. 


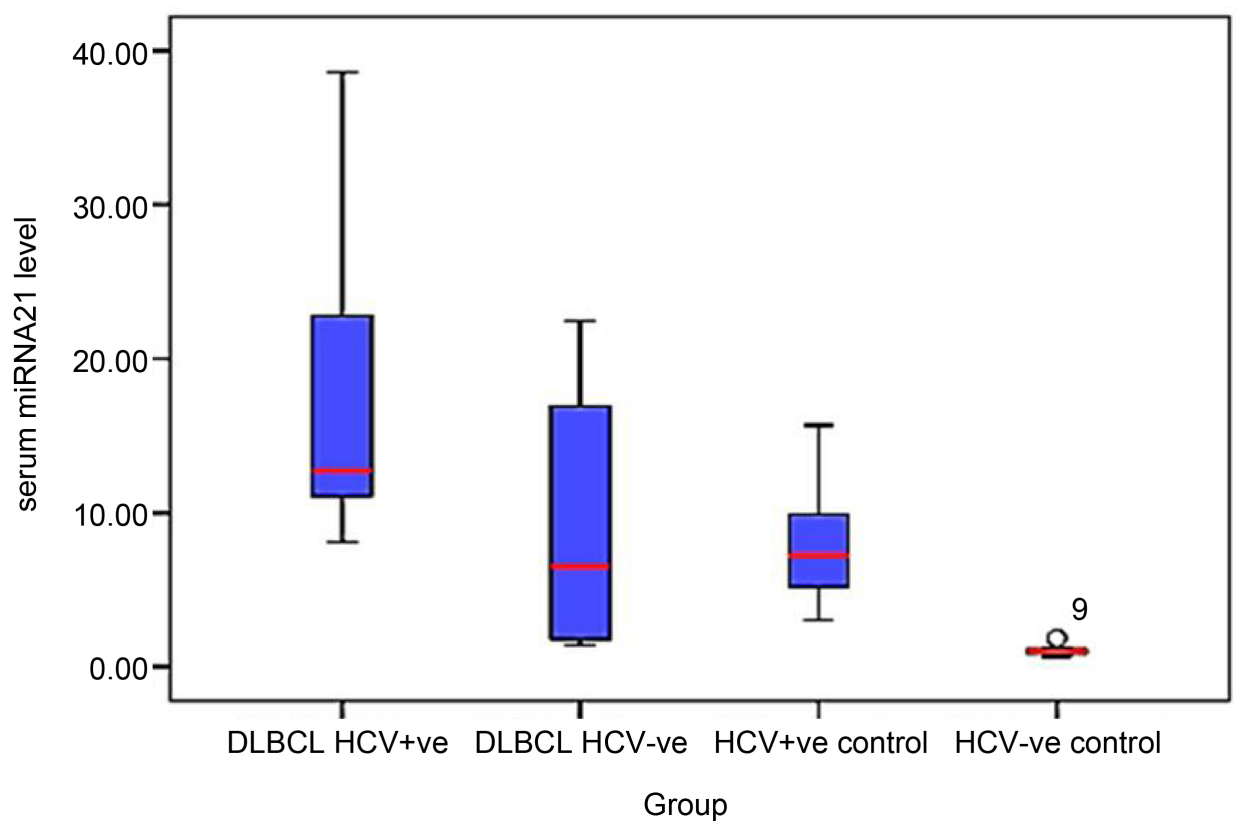

Figure 6. Comparison of miRNA-21 levels in sera of; HCV positive DLBCL patients, HCV negative DLBCL patients, HCV positive controls \& HCV negative controls. Highest levels of miRNA-21 were detected in HCV positive DLBCL patients $(n=10)$, followed by HCV negative DLBCL $(n=$ $20)$, then HCV positive pathological controls $(n=10)$, with the lowest levels being detected in HCV negative controls $(n=10)$. The band inside each box plot is the median. The outlier's samples (those whose expression extends beyond 1.5 times the interquartile range) are represented as dots: the white dots are the middle outlier while the black stars are the extreme outlier.

Table 4. Correlations between serum miRNA-21expression and markers of clinical and prognostic influence in HCV positive DLBCL cases.

\begin{tabular}{ccc}
\hline & \multicolumn{2}{c}{ Serum miRNA-21 HCV positive DLBCL } \\
\cline { 2 - 3 } Parameter & $\mathrm{r}_{\mathrm{s}}$ & $\mathrm{p}$ \\
\hline IPI & 0.343 & 0.332 \\
Stage & 0.694 & $0.026^{*}$ \\
ECOG PS & 0.224 & 0.534 \\
Viral load & 0.547 & 0.102 \\
SGOT & 0.248 & 0.489 \\
SGPT & 0.152 & 0.676 \\
Total Bilirubin & 0.122 & 0.736 \\
Direct Bilirubin & 0.248 & 0.224 \\
Albumin & -0.535 & 0.489 \\
\hline
\end{tabular}

$\mathrm{r}_{\mathrm{s}}$ : Spearman rho correlation coefficient. *: Significant correlation.

Higher levels of miRNA-21 were detected in HCV positive ABC DLBCL patients ( $\mathrm{n}=$ $7,11.03$ to 38.58 , median; 14.62 ) compared to GCB patients. ( $\mathrm{n}=3,8.11$ to 11.63 , median; 10.2) with borderline statistical significance $(\mathrm{U}=2.000, \mathrm{p}=0.058)$.

- Serum miRNA-21 expression levels compared in HCV positive DLBCL cases in relation to response to treatment. 
Patients with no response to therapy $(n=3)$ expressed the highest serum miRNA-21 levels which ranged from 10.20 to 38.58 with a median of 30.48 . In patients who achieved CR $(\mathrm{n}=4)$ miRNA-21 level ranged from 8.11 to 13.83 with a median of 11.36 , while, in patients who achieved PR $(n=3)$ miRNA-21 ranged from 11.56 to 22.78 with a median of 14.62. The difference in miRNA-21 expression between the three groups was not statistically significant (Chi square $=2.300, \mathrm{p}=0.317$ ).

\section{- MiRNA-21 level in control group.}

Serum miRNA-21 expression levels were compared in HCV positive and HCV negative control groups. Significantly higher levels were detected in HCV positive controls ( $\mathrm{n}=10,1.41$ to 22.47 , median; 6.515$)$ compared to HCV negative controls $(\mathrm{n}=10,0.7$ to 1.87 , median 1.0135$)$. $(\mathrm{U}=3.000$, p value $=0.000)$.

In $\mathrm{HCV}$ positive controls, serum miRNA-21 level was correlated with a number of parameters; viral load, SGOT, SGPT, ALP, total bilirubin, direct bilirubin, prothrombin activity and albumin using spearman's correlation test. MiRNA-21 level showed statistically significant positive correlation with SGOT $\left(r_{s}=0.636, p=0.048\right), \operatorname{SGPT}\left(r_{s}=\right.$ $0.685, \mathrm{p}=0.029), \operatorname{ALP}\left(\mathrm{r}_{\mathrm{s}}=0.636, \mathrm{p}=0.048\right)$, total bilirubin $\left(\mathrm{r}_{\mathrm{s}}=0.790, \mathrm{p}=0.007\right)$, direct bilirubin $\left(r_{s}=0.766, p=0.010\right)$ and the viral load $\left(r_{s}=0.891, p=0.001\right)$. A statistically significant negative correlation between miRNA-21 and serum albumin was detected $\left(\mathrm{r}_{\mathrm{s}}=-0.827, \mathrm{p}=0.003\right)$. Prothrombin activity showed a negative correlation with miRNA-21 that didn't reach statistical significance $\left(r_{s}=-0.283, p=0.428\right)($ Table 5).

\section{- Survival analysis.}

OS and PFS of DLBCL patients $(n=30)$ were calculated using Kaplan Meier survival curve. Total number of deaths from randomization till 24 month was 8 cases $(26.67 \%)$ and OS was $73.3 \%$, while total number of cases with disease progression or death from randomization till 24 month was 16 cases (53.33\%) with a PFS of $46.67 \%$. At 24 months follow up, ABC DLBCL patients showed worse OS (61.1\%) than GCB patients (91.7\%), but the difference was not statistically significant (chi square Log Rank $=3.057, \mathrm{p}=$ 0.080). Similarly, ABC subtype showed worse PFS (33.33\%) compared to GCB subtype $(66.67 \%)$ and difference was not statistically significant (chi square Log Rank $=2.975, \mathrm{p}$ $=0.085)$.

Table 5. Correlations between serum miRNA-21 levels and hepatic parameters in HCV positive control group.

\begin{tabular}{ccc}
\hline \multirow{2}{*}{ Parameter } & \multicolumn{2}{c}{ Serum miRNA-21 HCV positive control } \\
\cline { 2 - 3 } & $\mathrm{r}_{\mathrm{s}}$ & $\mathrm{p}$ \\
\hline Viral load & 0.891 & $0.001^{\star}$ \\
SGOT & 0.636 & $0.048^{\star}$ \\
SGPT & 0.685 & $0.029^{\star}$ \\
ALP & 0.636 & $0.048^{\star}$ \\
Total Bilirubin & 0.79 & $0.007^{\star}$ \\
Direct Bilirubin & 0.766 & $0.010^{\star}$ \\
Prothrombin activity & -0.283 & 0.428 \\
Albumin & -0.827 & $0.003^{\star}$ \\
\hline
\end{tabular}

$\mathrm{r}_{\mathrm{s}}$ : Spearman rho correlation coefficient. ${ }^{*}$ : Significant correlation. 
Using the median of serum miRNA-21, DLBCL patients were allocated into two groups. Patients with miRNA-21 level higher than median were assigned to the highexpression group, while those with levels lower than median were allocated to the lowexpression group. Higher expression levels of miRNA-21 were associated with significantly worse 24 months OS (53.33\%) compared to lower expression levels (93.33\%), (chi square $\log _{\text {Rank }}=5.703, \mathrm{p}=0.017$ ). Similarly, higher expression levels of miRNA-21 were associated with significantly worse 24 months PFS (26.67\%) compared to lower expression levels (73.33\%) (chi square Log Rank $_{1}=8.869, \mathrm{p}=0.003$ ) (Figure 7).

In HCV positive DLBCL cases $(\mathrm{n}=10)$ at 24 month follow up, OS was $80 \%$ and PFS was $40 \%$. In HCV negative DLBCL cases $(n=20)$, OS was $70 \%$, while the PFS was $50 \%$. Survival rates didn't differ between HCV positive and HCV negative DLBCL cases; OS \left.${\left.\text { (chi } \text { square }_{\text {Log Rank }}=0.282, \mathrm{p}=0.596\right) \text { and PFS (chi square }}_{\text {Log Rank }}=0.037, \mathrm{p}=0.848\right)$.

\section{Discussion}

MiRNA-21 is one of the most frequently studied cancer related miRNAs [21]. In the field of hematological malignancies the prognostic impact of miRNA-21 has been evaluated in various leukemias and lymphomas. Acting as an oncogene, elevated miRNA-21

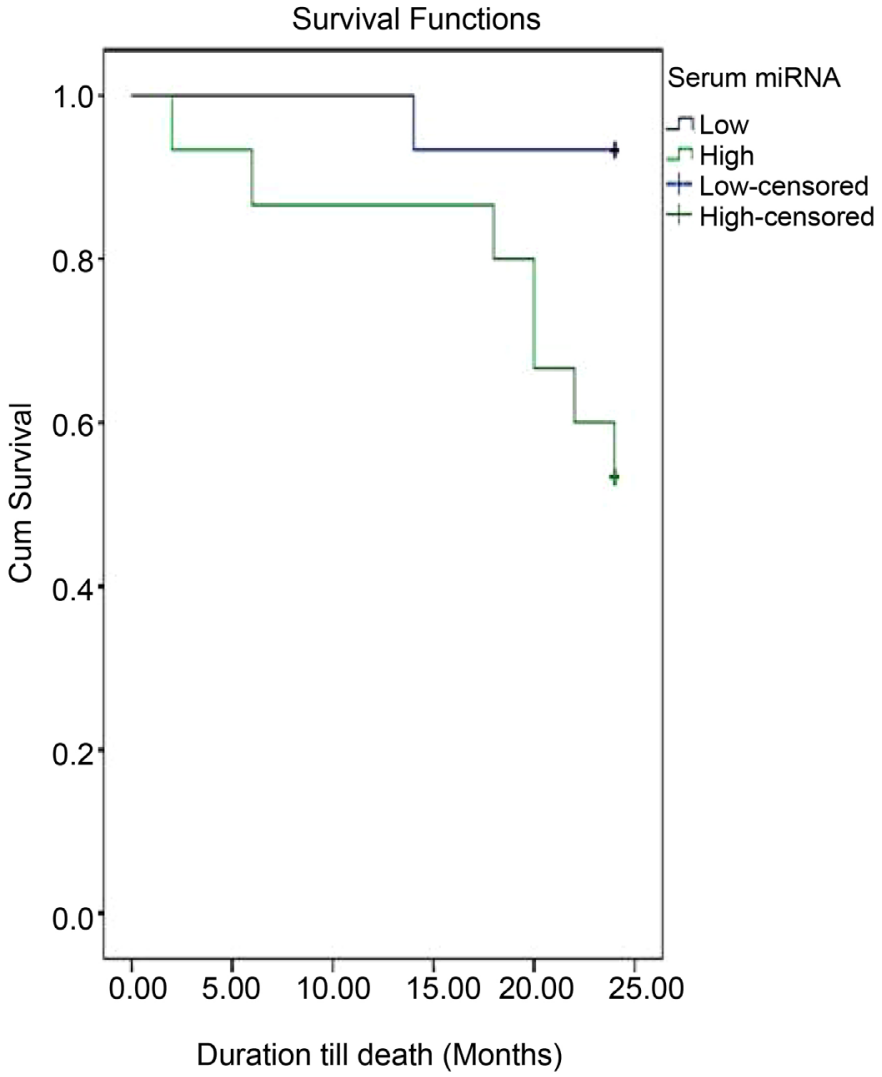

(A)

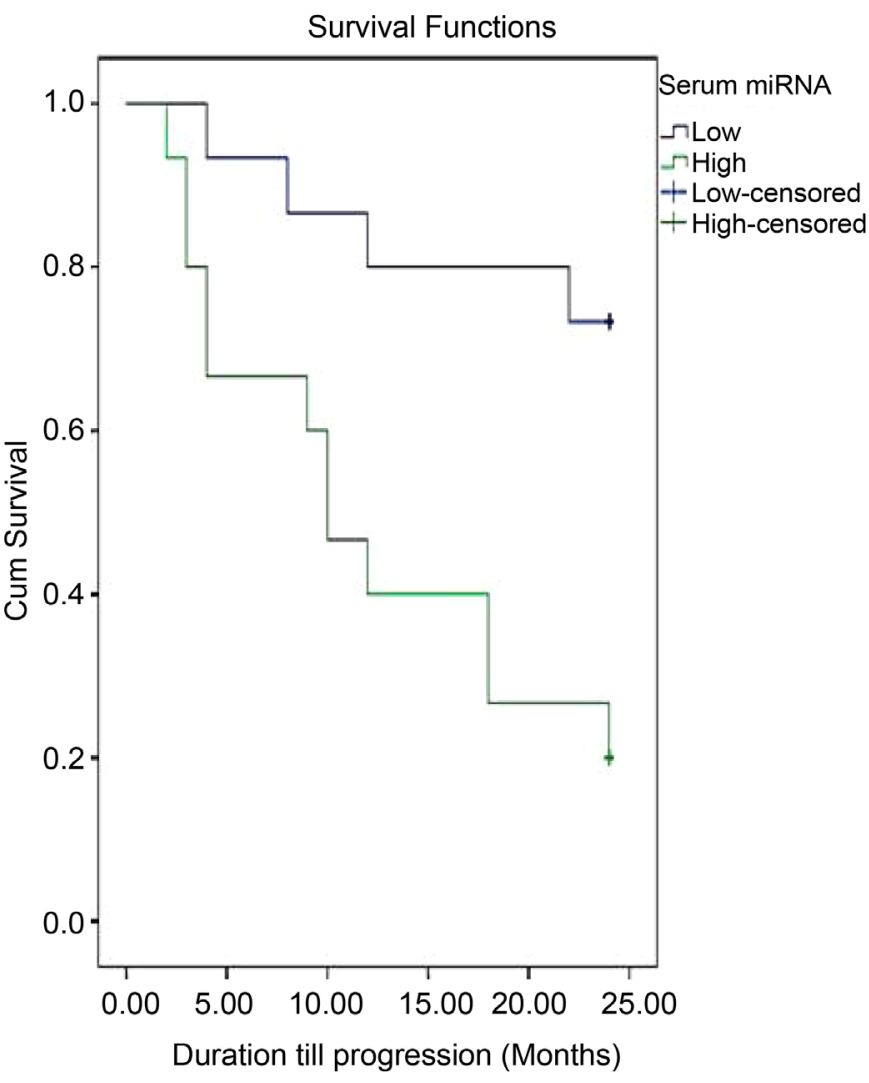

(B)

Figure 7. The impact of miRNA-21 expression on survival of DLBCL patients. Kaplan Meier survival curves showing (A) OS and (B) PFS were compared by univariate (log rank) analysis. MiRNA-21 expression levels in patients $(\mathrm{n}=30)$ were defined as high or low relative to the median. Higher levels were associated with worse prognosis in terms of OS and PFS in DLBCL patients. 
alone was found to be sufficient to induce a pre-B malignant lymphoid-like phenotype in animal models, and its ongoing expression was essential to maintain the tumor phenotype [22].

MiRNA-21 has been considered a negative prognostic biomarker, being associated with tumor aggressiveness and poor outcome [21]. The prognostic value of miRNA-21 and its role in predicting the response to treatment and survival in DLBCL with its two molecular subtypes have been evaluated in the current study, which displayed a higher miRNA-21 over-expression in DLBCL patients (3.03 to 38.53, median; 9.32) compared to controls ( 0.7 to 22.47 median; 1.44 ) with as statistically significant result. Similarly, a higher serum miRNA-21 level was detected in patients with $A B C$ sub-type (5.82 to 38.58, median; 11.44) compared to GCB sub-type (3.03 to 11.63 , median; 5.67 ) with a statistically significant difference between the two groups. This finding coincides with the results of Lawrie CH et al. (2008) [10], Chen W et al. (2014) [9], Li J et al. (2015) [16] who studied miRNA-21 expression in sera of DLBCL patients as well as the results of Li CH et al. (2014) [23], Fu R et al. (2014) [24], and Zajdel M et al. (2015) [25] who studied the expression of the same miRNA on paraffin embedded tissue specimens.

MiRNA-21 expression was compared in relation to immunohistochemical markers of DLBCL. A significantly higher miRNA-21 expression was detected in cases negative for BCL6 compared to cases which express BCL6. Similarly, a significantly higher miRNA-21 expression was detected in CD10 negative, as well as MUM1 positive cases, compared to CD10 positive and MUM1 negative groups. Comparing miRNA-21 expression in relation to BCL2, higher levels were detected in cases positive for BCL2 compared BCL2 negative cases, but the result didn't reach statistical significance.

Regarding the prognostic value of miRNA-21 in DLBCL, we found that higher miRNA-21 expression levels were associated with poor response to chemotherapy, with a statistically significant result. Serum miRNA-21 in patients who achieved CR showed moderate over expression (median; 6.44). In contrast, in patients who failed to respond to treatment miRNA-21 level disclosed marked over expression (11.845). Higher expression levels were associated with a significantly worse OS and PFS. Patients with lower miRNA-21 over-expression showed an OS of 93.33\%, while patients with higher levels showed an OS of $53.33 \%$. Similarly, patients with lower miRNA-21 over expression showed a PFS of $73.33 \%$ while in patients with higher levels PFS was $20 \%$.

Our results coincides with the results reported by Li J (2015) [16], confirming the worse overall survival associated with higher expression of miRNA-21. In contradiction with the current results, Lawrie CH et al. (2008) [10] as well as Chen W (2014) [9] reported that higher expression levels of miRNA-21 in sera of DLBCL patient were found to be associated with improved PFS. The study of Munch-Peterson HD et al. (2015) revealed that miRNA-21 detection in cases of DLBCL did not add prognostic information and that it acts mainly as an inflammatory marker expressed by the bystander cells [26]. On the other hand, Roehle A et al., (2008) reported that a poor OS in DLBCL patients was most strongly correlated with down regulated expression of miRNA-21 [27].

In addition, the present study also disclosed significant correlations between miRNA-21 and some of the well-known markers of clinical and prognostic implications in DLBCL. Statistically significant positive correlations were detected between serum 
miRNA-21 and serum LDH, IPI and the disease stage. Correlations between miRNA-21 and the age, ESR and ECOG PS were not statistically significant. In GCB-DLBCL cases, miRNA-21 expression didn't show a significant difference between early (I/II) and advanced (III/IV) disease stage. On the other hand, in ABC-DLBCL cases statistically significant higher miRNA-21 levels were detected in advanced disease stage compared to early disease stage. This is in agreement with $\mathrm{Li} \mathrm{CH}$ et al. (2014) [23] who reported a positive correlation between miRNA-21 expression levels and serum LDH as well as the Ann Arbor stage, with a higher miRNA-21 level in stage III-IV compared to stage I-II. Li J et al. (2015) reported [16] a significantly positive correlation between serum miRNA-21 level and the IPI, which coincides with the current results. In contrast, Lawrie C's study (2008) [10] didn't reveal any significant correlations between miRNA-21 levels and clinicopathological features of the DLBCL patients; including the IPI and disease stage, while Chen W et al. (2014) [9] reported that serum miRNA-21 levels in DLBCL patients in stage I and II were significantly higher than those patients in stage III and IV in both subtypes.

Moreover, in the present study we performed a detailed assessment of the differential expression of miRNA-21 in the two molecular subtypes of DLBCL and correlated it with clinical findings as well as markers of prognostic significance in each subtype. In GCB cases, a higher miRNA-21 was detected in BCL2 positive cases than BCL2 negative cases but the difference didn't reach statistical significance. In ABC like DLBCL cases, miRNA-21 was almost the same in BCL2 positive and negative cases There was no significant difference in miRNA-21 expression in cases presenting with B symptoms and cases with evidence of BM infiltration compared to cases without such findings in each sub-type. MiRNA-21 over-expression was higher in GCB patients with ECOG PS $\geq 2$ than in cases with ECOG 0-1 but the difference was not statistically significant, while in $\mathrm{ABC}$ patients the levels were almost the same in both ECOG PS sub-groups.

A higher miRNA-21 level was detected in ABC cases with advanced stage (III/IV) than in cases with early stage (I/II) with statistical significance. In GCB-DLBCL cases miRNA-21 levels in advanced stage (III/IV) were quite similar to its levels in early stage (I/II). Comparing miRNA-21 level between different IPI scores in GCB and ABC subgroups, we found no statistically significant difference in either sub-groups.

In addition, we found that higher miRNA-21 expression was associated with poor response to treatment in each sub-type of DLBCL, with the highest levels being detected in patients with no response (GCB; median 7.46 and $A B C ; 14.22$ ) and the lowest level being detected in patients with CR ( GCB; median 4.76 and ABC; 7.41). The difference was not statistically significant in GCB sub-type but showed borderline statistical significance in $A B C$ sub-type. Though in most of the examined parameters ABC sub-type was associated with worse prognosis, failure to reach statistical significance may be attributed to small sample size.

Over the past two decades there has been a large sum of published studies from different regions on the association between HCV and lymphomas [28]. Multiple studies also investigated the relation between miRNA expression and chronic HCV infection, hepatic fibrosis and cirrhosis [29] [30]. However, regarding the expression of miRNA-21 in HCV associated DLBCL to our knowledge there is no existing data so far. In the present study, we investigated the relation between $\mathrm{HCV}$ infection and serum 
miRNA-21 expression in DLBCL. Correlations between miRNA-21 and different hepatic parameters in $\mathrm{HCV}$ positive patients as well as $\mathrm{HCV}$ positive controls were also analyzed.

We compared serum miRNA-21 level in 4 groups; HCV positive DLBCL patients, HCV negative DLBC patients, HCV positive pathological controls and HCV negative healthy controls. A highly statistically significant difference in miRNA-21 expression was detected between the four groups, with the highest expression level being detected in HCV positive lymphoma patients (median; 12.73). Lower levels were detected in HCV negative lymphoma patients (median; 7.235) and HCV positive pathological controls (median; 6.515) with the lowest level being detected in HCV negative healthy controls (median; 1.0135).

In HCV positive DLBCL serum miRNA-21 was correlated with different hepatic parameters. Only the disease stage showed statistically significant positive correlation with miRNA-21 while all other parameters; IPI, ECOG PS, viral load, total bilirubin, direct bilirubin, SGOT, SGPT and HCV prognostic score showed positive correlation with miRNA-21 level which failed to reach statistical significance. Serum albumin showed a negative correlation with miRNA-21 which also failed to reach statistical significance. Regarding the differential expression of miRNA-21 in the two molecular sub-types in HCV associated DLBCL, higher miRNA-21 levels were detected in HCV positive ABC sub-type (median; 14.62) compared to GCB sub-type (median; 10.2), with the result showing borderline statistical significance.

Serum miRNA-21 in HCV patients who achieved CR (40\%) ranged from 8.11 to 13.83 with a median of 11.36 , while, in PR group (30\%) it ranged from 11.56 to 22.78 with a median of 14.62 and in the patients who showed no response $(30 \%)$ it ranged from 10.20 to 38.58 with a median of 30.48 . Higher miRNA-21 expression levels were associated with poor response to treatment; however the result didn't reach statistical significance. Regarding the 2 years survival analysis, in HCV positive DLBCL cases, the OS was $80 \%$ and PFS was $40 \%$, while in the HCV negative DLBCL cases the OS was $70 \%$ and PFS of $50 \%$ with no significant difference between the two groups. Failure to achieve statistical significance in most of the correlation of miRNA-21 in HCV positive DLBCL patients may be attributed to small sample size.

The expression levels of miRNA-21 in sera of HCV positive subjects without lymphoma, compared to its levels in sera of HCV negative completely healthy controls was compared in the current study in an attempt to display the clinical impact and prognosis of miRNA-21 expression as a marker of necro-inflammation, in such category of patients with one of the most common endemic health issues in our country; Egypt. The results revealed a significantly higher serum miRNA-21 in HCV positive pathological controls (median; 6.515) compared to HCV negative controls (median; 1.0135). Serum miRNA-21 in HCV positive controls showed significant positive correlations with SGOT, SGPT, and viral load, ALP, total bilirubin and direct bilirubin. Significant negative correlation between miRNA-21 and serum albumin was also detected.

Our findings coincide with the results of the study performed at Lowa University by Marquez RT and colleagues [29], who reported that miRNA-21 was significantly elevated in $\mathrm{HCV}$ positive liver biopsies, compared to HCV negative samples and that 
miRNA-21 positively correlated with liver transaminases and viral load but not the genotype or age. Similar results were reported by Bihrer V et al. (2011) [31] who demonstrated that miRNA-21 level was increased in sera of patients with chronic HCV infection compared to control and positively correlated with ALT, AST, total bilirubin, INR, and $\gamma$-glutamyltransferase with statistical significance and negatively correlated with serum albumin level. In contradiction, with the current results, the study of Cermelli $S$ et al. (2011) [32] displayed that no significant correlations between miRNA-21 and serum transaminases, the fibrotic stage, the degree of inflammatory activity and HCV viral load could be detected.

In a recent study conducted in Egypt, Harfoush R, et al. (2016) [33] reported a statistically significant elevation in expression of miRNA-21 in plasma of chronic non cirrhotic HCV patients in comparison to cirrhotic HCV and to HCC groups as well as healthy controls. The investigators reported that miRNA-21showed a significant fold decrease in cirrhotic and HCC groups and suggested that serum miRNA-21 level may initially rise following release from inflamed hepatocytes in HCV infection followed by a drop in its levels with fibrosis progression due to hepatocyte loss and accumulation of extracellular matrix. In contradiction with the current study, as well as Bihrer $\mathrm{V}$ and Marquez RT's studies, the investigators reported significantly positive correlations between miRNA-21 levels and serum albumin as well as prothrombin activity, and significantly negative correlations with ALT, AST, total bilirubin, and ALP levels. There was no correlation between the concentrations of miRNA-21 and HCV viral load, which contradicts with the current results. We recommend further research in that field on a larger scale of HCV patients, considering detailed analysis of the relation of miRNA-21 expression with the degree of necro-inflammation and the stages of hepatic cirrhosis.

\section{Conclusion}

In summary our results suggest that miRNA-21 is a marker of diagnostic and prognostic relevance in DLBCL, with its two molecular subtypes. MiRNA-21 was overexpressed in DLBCL patients with higher levels detected in ABC patients compared to GCB patients. Higher miRNA-21 levels were associated with poor response to treatment and a worse 2-year OS and PFS in DLBCL. To our knowledge, the present study is the first to investigate the expression of miRNA-21 in HCV related DLBCL. Higher miRNA-21 levels were detected in HCV positive DLBCL patients compared to HCV negative DLBCL patients as well as controls with HCV infection and healthy controls. Higher levels were also detected in HCV positive ABC DLBCL patients compared to GCB sub-type. Hence our study shows that miRNA-21 is a potential marker of necro-inflammation in addition to its role in tumorogenesis. We hope that the current results would be a starting point for more research in circulating miRNAs on larger samples of patients with HCV associated DLBCL.

\section{Conflicts of Interest}

The authors hereby declare that they have no conflicts of interest. This research was completely funded by the authors. All the authors of this paper have no financial relationship with the organization where the research was carried out. 


\section{References}

[1] De Tullio, G., De Fazio, V., Sgherza, N., Minoia, C., Serratì, S., Merchionne, F., et al. (2014) Challenges and Opportunities of MicroRNAs in Lymphomas. Molecules, 19, 14723-14781. https://doi.org/10.3390/molecules190914723

[2] De Yébenes, V.G., Bartolomé-Izquierdo, N. and Ramiro, A.R. (2013) Regulation of B-Cell Development and Function by MicroRNAs. Immunological Reviews, 253, 25-39. https://doi.org/10.1111/imr.12046

[3] Tili, E., Michaille, J.J. and Croce, C.M. (2013) MicroRNAs Play a Central Role in Molecular Dysfunctions Linking Inflammation with Cancer. Immunological Reviews, 253, 167-184. https://doi.org/10.1111/imr.12050

[4] Fu, X., Han, Y., Wu, Y., Zhu, X., Lu, X., Mao, F., et al. (2011) Prognostic Role of MicroRNA-21 in Various Carcinomas: A Systematic Review and Meta-Analysis. European Journal of Clinical Investigation, 41, 1245-1253. https://doi.org/10.1111/j.1365-2362.2011.02535.x

[5] Xia, X., Yang, B., Zhai, X., Liu, X. Shen, K., Wu, Z., et al. (2013) Prognostic Role of MicroRNA-21 in Colorectal Cancer: A Meta-Analysis. PLoS ONE, 8, e80426. https://doi.org/10.1371/journal.pone.0080426

[6] Hu, G., Tao, F., Wang, W. and Ji, K. (2016) Prognostic Value of MicroRNA-21 in Pancreatic Ductal Adenocarcinoma: A Meta-Analysis. World Journal of Surgical Oncology, 14, 82. https://doi.org/10.1186/s12957-016-0842-4

[7] Wu, K., Li, L. and Li, S. (2015) Circulating MicroRNA-21 as a Biomarker for the Detection of Various Carcinomas: an Updated Meta-Analysis Based on 36 Studies. Tumor Biology, 36, 1973-1981. https://doi.org/10.1007/s13277-014-2803-2

[8] Lawrie, C.H., Soneji, S., Marafioti, T., Cooper, C., Palazzo, S., Paterson, J.C., et al. (2007) MicroRNA Expression Distinguishes between Germinal Center B Cell-Like and Activated B Cell-Like Subtypes of Diffuse Large B Cell Lymphoma. International Journal of Cancer, 121, 1156-1161. https://doi.org/10.1002/ijc.22800

[9] Chen, W., Wang, H., Chen, H., Liu, S., Lu, H., Kong, D., et al. (2014) Clinical Significance and Detection of MicroRNA-21 in Serum of Patients with Diffuse Large B-Cell Lymphoma in Chinese Population. European Journal of Haematology, 92, 407-412. https://doi.org/10.1111/ejh.12263

[10] Lawrie, C.H., Gal, S., Dunlop, H.M., Pushkaran, B., Liggins, A.P., Pulford, K., et al. (2008) Detection of Elevated Levels of Tumour-Associated MicroRNAs in Serum of Patients with Diffuse Large B-Cell Lymphoma. British Journal of Haematology, 141, 672-675. https://doi.org/10.1111/j.1365-2141.2008.07077.x

[11] Go, H., Jang, J.Y., Kim, P.J., Kim, Y.G., Nam, S.J., Paik, J.H., et al. (2015) MicroRNA-21 Plays an Oncogenic Role by Targeting FOXO1 and Activating the PI3K/AKT Pathway in Diffuse Large B-Cell Lymphoma. Oncotarget, 6, 15035-15049. https://doi.org/10.18632/oncotarget.3729

[12] Bai, H., Wei, J., Deng, C., Yang, X., Wang, C. and Xu, R. (2013) MicroRNA-21 Regulates the Sensitivity of Diffuse Large B-Cell Lymphoma Cells to the CHOP Chemotherapy Regimen. International Journal of Hematology, 97, 223-231. https://doi.org/10.1007/s12185-012-1256-x

[13] Gravitz, L. (2011) Introduction: A Smouldering Public-Health Crisis. Nature, 474, S2-S4. https://doi.org/10.1038/474S2a

[14] Kanwal, F., Hoang, T., Kramer, J.R., Asch, S.M., Goetz, M.B., Zeringue, A., et al. (2011) Increasing Prevalence of HCC and Cirrhosis in Patients with Chronic Hepatitis C Virus Infection. Gastroenterology, 140, 1182-1188. https://doi.org/10.1053/j.gastro.2010.12.032

[15] Motawi, T.K., Shaker, O.G., El-Maraghy, S.A. and Senousy, M.A. (2015) Serum MicroRNAs 
as Potential Biomarkers for Early Diagnosis of Hepatitis C Virus-Related Hepatocellular Carcinoma in Egyptian Patients. PLoS ONE, 10, e0137706. https://doi.org/10.1371/journal.pone.0137706

[16] Li, J., Fu, R., Yang, L. and Tu, W. (2015) MiR-21 Expression Predicts Prognosis in Diffuse Large B-Cell Lymphoma. International Journal of Clinical and Experimental Pathology, 8, 15019-15024.

[17] Peveling-Oberhag, J., Crisman, G., Schmidt, A., Döring, C., Lucioni, M., Arcaini, L., et al. (2012) Dysregulation of Global MicroRNA Expression in Splenic Marginal Zone Lymphoma and Influence of Chronic Hepatitis C Virus Infection. Leukemia, 26, 1654-1662. https://doi.org/10.1038/leu.2012.29

[18] Swerdlow, S.H., Campo, E., Harris, N.L., Jaffe, E.S., Pileri, S.A., Stein, H., et al. (2008) WHO Classification of Tumours of Haematopoietic and Lymphoid Tissues. 4th Edition, IARC Press, Lyon, 2.

[19] Swerdlow, S.H., Campo, E., Pileri, S.A., Harris, N.L., Stein. H., Siebert, R., et al. (2016) The 2016 Revision of the World Health Organization Classification of Lymphoid Neoplasms. Blood, 19, 127, 2375-2390.

[20] Hans, C.P., Weisenburger, D.D., Greiner, T.C., Gascoyne, R.D., Delabie, J., Ott, G., et al. (2004) Confirmation of the Molecular Classification of Diffuse Large B-Cell Lymphoma by Immunohistochemistry Using a Tissue Microarray. Blood, 103, 275-282. https://doi.org/10.1182/blood-2003-05-1545

[21] Krichevsky, A.M. and Gabriely, G. (2009) MiR-21: A Small Multi-Faceted RNA. Journal of Cellular and Molecular Medicine, 13, 39-53. https://doi.org/10.1111/j.1582-4934.2008.00556.x

[22] Medina, P.P., Nolde, M. and Slack, F.J. (2010) OncomiR Addiction in an in Vivo Model of MicroRNA-21-Induced Pre-B-Cell Lymphoma. Nature, 467, 86-90. https://doi.org/10.1038/nature09284

[23] Li, C.H., Fu, R., Wang, Y.H., Song, W.J. and Ruan, E.B., et al. (2014) Expression and Clinical Significance of MiR-21 in Diffuse Large B Cell Lymphoma. Journal of Experimental Hematology, 22, 339-343.

[24] Fu, R., Li, C. and Shao, Z. (2014) Aberrant Over Expression and Regulatory Mechanism of Mir-21 in Diffuse Large B Cell Lymphoma. Blood, 124, 5171-5175.

[25] Zajdel, M., Rymkiewicz, G., Chechlinska, M., Blachnio, K. and Pienkowska-Grela, B., Grygalewicz, B., et al. (2015) MiR Expression in MYC-Negative DLBCL/BL with Partial Trisomy 11 Is Similar to Classical Burkitt Lymphoma and Different from Diffuse Large B-Cell Lymphoma. Tumor Biology, 36, 5377-5388. https://doi.org/10.1007/s13277-015-3203-y

[26] Munch-Petersen, H.D., Ralfkiaer, U., Sjö, L.D., Hother, C., Asmar, F., Nielsen, B.S., et al. (2015) Differential Expression of MiR-155 and MiR-21 in Tumor and Stroma Cells in Diffuse Large B-Cell Lymphoma. Applied Immunohistochemistry \& Molecular Morphology, 23, 188-195. https://doi.org/10.1097/PAI.0000000000000073

[27] Roehle, A., Hoefig, K.P., Repsilber, D., Thorns, C., Ziepert, M., Wesche, K.O., et al. (2008) MicroRNA Signatures Characterize Diffuse Large B-Cell Lymphomas and Follicular Lymphomas. British Journal of Haematology, 142, 732-744. https://doi.org/10.1111/j.1365-2141.2008.07237.x

[28] Vannata, B., Arcaini, L. and Zucca, E. (2016) Hepatitis C Virus-Associated B-Cell NonHodgkin's Lymphomas: What Do We Know? Therapeutic Advances in Hematology, 7, 94107. https://doi.org/10.1177/2040620715623924

[29] Marquez, R.T., Bandyopadhyay, S., Wendlandt, E.B., Keck, K., Hoffer, B.A., Icardi, M.S., et al. (2010) Correlation between MicroRNA Expression Levels and Clinical Parameters Associated with Chronic Hepatitis C Viral Infection in Humans. Laboratory Investigation, 90, 1727-1736. https://doi.org/10.1038/labinvest.2010.126 
[30] Gragnani, L., Piluso, A., Fognani, E and Zignego, A.L. (2015) MicroRNA Expression in Hepatitis C Virus-Related Malignancies: A Brief Review. World Journal of Gastroenterology, 21, 8562-8568. https://doi.org/10.3748/wjg.v21.i28.8562

[31] Bihrer, V., Waidmann, O., Friedrich-Rust, M., Forestier, N., Susser, S.,Haupenthal, J., et al. (2011) Serum MicroRNA-21 as Marker for Necroinflammation in Hepatitis C Patients with and without Hepatocellular Carcinoma. PLOS ONE, 6, e26971. https://doi.org/10.1371/journal.pone.0026971

[32] Cermelli, S., Ruggieri, A., Marrero, J.A., Ioannou, G.N. and Beretta, L. (2011) Circulating MicroRNAs in Patients with Chronic Hepatitis C and Non-Alcoholic Fatty Liver Disease. PLoS ONE, 6, e23937. https://doi.org/10.1371/journal.pone.0023937

[33] Harfoush, R., Meheissen, M., Elwafa, R. and Elwazzan, D. (2016) The Role of Circulating MicroRNAs as Markers of Disease Progression in Hepatitis C Virus Infected Egyptian Patients. Advances in Microbiology, 6, 320-331. https://doi.org/10.4236/aim.2016.64031

Submit or recommend next manuscript to SCIRP and we will provide best service for you:

Accepting pre-submission inquiries through Email, Facebook, LinkedIn, Twitter, etc. A wide selection of journals (inclusive of 9 subjects, more than 200 journals)

Providing 24-hour high-quality service

User-friendly online submission system

Fair and swift peer-review system

Efficient typesetting and proofreading procedure

Display of the result of downloads and visits, as well as the number of cited articles

Maximum dissemination of your research work

Submit your manuscript at: http://papersubmission.scirp.org/

Or contactajmb@scirp.org 